\title{
Vibration attenuation by a combination of a piezoelectric stack and a permanent magnet
}

\author{
A. Nandi ${ }^{\mathrm{a}, *}, \mathrm{~S} . \mathrm{Neogy}^{\mathrm{a}}, \mathrm{S}$. Bhaduri $^{\mathrm{b}}$ and H. Irretier ${ }^{\mathrm{c}}$ \\ ${ }^{a}$ Department of Mechanical Engineering, Jadavpur University, Kolkata, India \\ ${ }^{\mathrm{b}}$ Department of Applied Mechanics, Birla Institute of Technology, Mesra Ranchi, India \\ ${ }^{\mathrm{c}}$ Institute for Mechanics, University of Kassel, Kassel, Germany
}

Received 20 April 2011

Revised 12 October 2011

\begin{abstract}
The present work proposes a non-contact vibration attenuator made up of a permanent magnet mounted on a piezoelectric stack. Two such actuators are made to work simultaneously in a 'twin-actuator' configuration. It is conceived that a controlled change in the gap between the actuator and the structure is capable of attenuation of vibration of the structure. This appropriate change in gap is achieved by controlled motion of the piezoelectric stacks. It is shown that the actuator works as an active damper when the extension and contraction of the actuators are made proportional to the velocity of the beam. The resolution of extension of a piezoelectric stack is in the order of nanometers. Thus in the proposed actuator the force of actuation can be applied with great precision. This actuator is also attractive for its simple constructional feature.
\end{abstract}

Keywords: Piezoelectric stack, permanent magnet, active damper, non-contact actuator, non-linear simulation

\section{Introduction}

Magnetic and piezoelectric stack actuators have been extensively used for vibration excitation and control of structures and rotors.

Schweitzer et al. [11], Genta [2], Chiba et al. [1] systematically documented the extensive research output in the field of active magnetic bearings in their well-known texts on the subject.

Kreuzinger-Janik and Irretier [5,6] have utilized electromagnetic actuators for vibration excitation in modal analysis of rotors. They have developed a one-sided magnetic exciter for this purpose and used it for determination of modal parameters of rotors up to higher modes.

Recently, Strohschein and Irretier [13] developed an electromagnetic damper for vibration control of a flexible test rotor. This electromagnetic damper provides damping without levitation. It has a very compact construction and contains twin electromagnets, laser displacement sensors and piezoelectric force sensors in a single housing. The whole arrangement can be shifted along the length of the rotor and placed in a position most effective from the point of view of vibration reduction. The shape of the E-shaped electromagnets is modified in an innovative way to strengthen the magnetic field and increase the resulting damping force.

Przybylowicz [10] considered a rigid rotor on journal bearings. The bearing shells are placed on identical piezoelectric stacks. Two perpendicular inductive sensors are used to measure the displacement of the journal. This displacement is differentiated and the piezoelectric stacks are made to move using a velocity feedback. Therefore the journal now spins and moves within a moving bearing shell. Under such conditions the governing equations

*Corresponding author: Department of Mechanical Engineering, Jadavpur University, Kolkata 700 032, India. E-mail: arghyan@ yahoo.com; anandi@mech.jdvu.ac.in. 
of motion for the rotor are derived. The resulting equations show an increase in the critical speed and the stability threshold.

Lee [7] reviewed the status of mechatronic systems in rotor dynamics. He discussed about electromagnetic dampers, one-sided magnetic actuators and piezoelectric stack actuators

Simoes et al. [12] devised an active bearing by a combination of a standard roller bearing and a piezoelectric stack. The roller bearing is held in a cage which is attached to a fixed chassis by means of flexible beams. This allows relative motion between the cage and the chassis. One end of a piezoelectric stack is fixed to the chassis. An axially stiff stem connects the stack to the cage. When a voltage is applied to the piezoelectric stack it tries to extend and in this way it applies a force on the bearing cage. The rotor vibrations are minimized using this actuator and a modal optimal control technique.

For the purpose of active vibration control, Kaletsch et al. [4] explored the possibility of replacing standard squeeze film dampers by active piezoelectric actuators. They performed a numerical study and an experimental investigation on the rotor stand in their institute. As piezoelectric actuators are weak in shear they have discussed about the need of shear load protection. They have also addressed the problem of overheating of the actuators.

The present work proposes a device, which combines a piezoelectric stack and a permanent magnet for non-contact vibration attenuation of beams and structures. Such a configuration has not been reported in literature. A simple control system has also been proposed to work with the above-mentioned actuator.

Once the proposed actuator works for a beam it can be adopted for rotor dynamic applications. To increase the force of actuation, the proposed actuator uses a couple of permanent magnets. One permanent magnet is attached to the structure being excited. The other one is placed on a support, set into motion by a piezoelectric stack. The second magnet and the piezoelectric stack, supporting it, constitute the proposed actuator. The present actuator operates on the principle of repulsion of like poles of permanent magnets. The magnet of the actuator has to be placed just below (or above) the magnet attached to the beam or structure being excited. When a voltage is applied to the piezoelectric stack it changes its length. As the piezoelectric stack changes its length, the gap between the actuator and the beam changes causing a force of actuation on the beam or structure being controlled.

The present work evaluates the performance of twin actuators of identical dimensions of the above-mentioned type for vibration damping of beams and structures. The twin actuators co-axially act either from the top and bottom or from the two sides. The arrangements of the poles are such that in static equilibrium condition both the actuators repel the beam.

Piezoelectric stacks are known for their nano-positioning capabilities. The resolution of movement of piezoelectric stack is in the order of nanometers. Therefore, the motion of the permanent magnet placed on the moving support and the resulting force of actuation can be very precisely controlled in the proposed exciter. Piezoelectric stacks generally have very high stiffness and natural frequency. This may enable one to neglect the dynamics of the stack in the frequency range of interest in mechanical vibration. The two main motivations for an attempt to analyze such an actuator are its expected capability of precise control of force and its simple constructional feature.

\section{Analysis}

Figure 1(a) shows a beam with twin actuators. This configuration will be referred to as the 'twin-actuator' in the rest of this document. The beam has magnets attached to it. The configuration of the poles of the magnets is shown in the above figure. There is repulsive force on the beam from both the actuators. In static condition, if forces from both the actuators are same, the beam is pushed towards a stable equilibrium position at the centre of the gap between the actuators. Figure 1(b) shows the displacement variables considered in the present work

In this section, the amount of magnetic force developed on a structure is first computed due to a single actuator. A simple model of a piezoelectric stack is described next. Finally, the equations of motion of a beam or structure along with twin-actuators are presented at the end. 


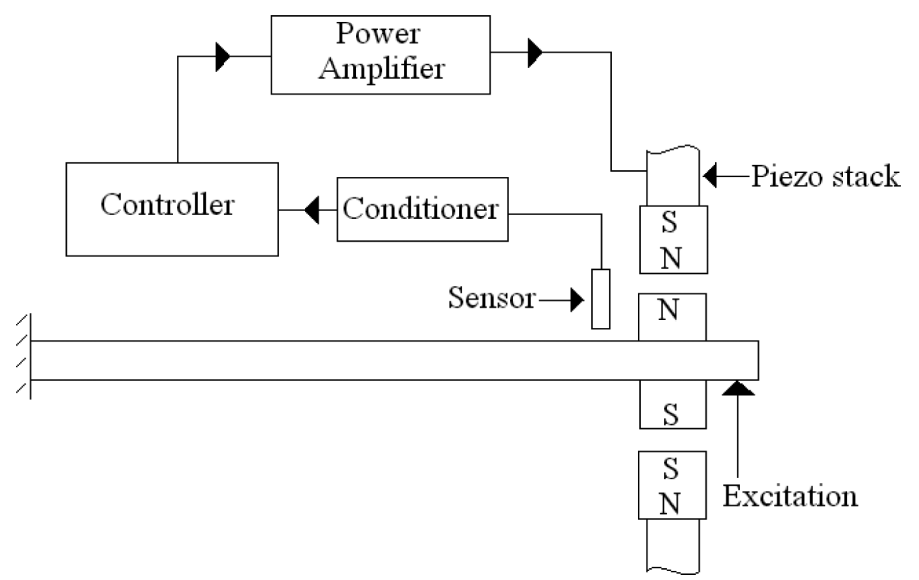

(a)

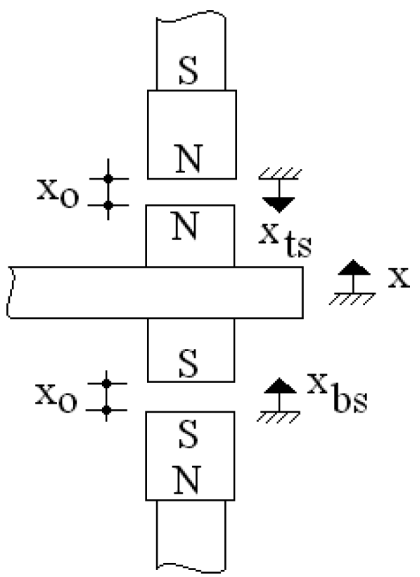

(b)

Fig. 1. (a). The arrangement of the beam along with twin actuators; (b). The gap and the displacements considered in the analysis.

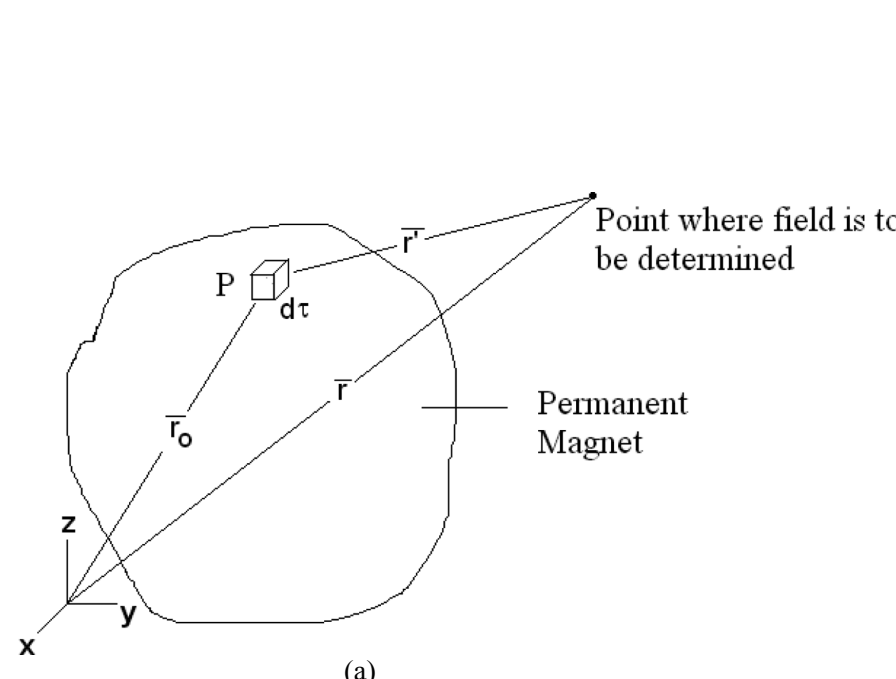

(a)

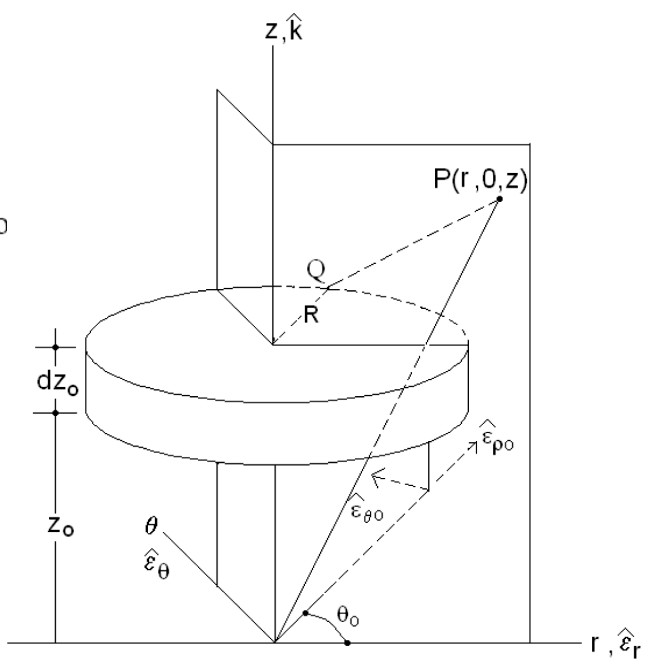

(b)

Fig. 2. (a). An infinitesimal volume in a permanent magnet and a point where field is sought; (b). Field due to an infinitesimal strip of permanent magnet.

\subsection{Force of actuation due to a single actuator}

For a single actuator, there are two repelling permanent magnets, one on the piezoelectric stack and the other on the beam or structure. The force of repulsion is computed as a function of the gap between the beam and the magnet. It is assumed here that the magnetization in both the permanent magnets is uniform and along the axial direction. Under such conditions both the magnets can be replaced by appropriate surface current densities [3]. The magnetic field due to the surface current of the actuator magnet can be computed using the well-known Biot-Savart law. This field generates a force on the surface current distribution of the permanent magnet attached to the beam or structure being excited. The details of the analysis are described below:-

\subsubsection{Determination of magnetic field generated by the actuator magnet}

The position vectors used in the subsequent analysis are explained in Fig. 2(a). The vector $\bar{r}_{o}$ denotes the position vector of a point $\mathrm{P}$ within the magnet. One requires determining the magnetic field at a position $\bar{r}^{\prime}$ relative to point 
P. Therefore, the field is to be determined at a position $\bar{r}=\bar{r}_{o}+\bar{r}^{\prime}$. A cylindrical magnetic material with uniform magnetization $\bar{M}\left(\bar{r}_{o}\right)$ along the axis of the cylinder is considered. If the $z$ axis is selected along the axis of the magnet the magnetization vector can be expressed as $\bar{M}=M \hat{k}$. It can be shown [3] that the field of this magnet is same as that generated by a surface current distribution $\bar{K}_{b}=\bar{M} \times \hat{n}$, where the symbol $\hat{n}$ stands for unit vector normal to the surface. For an infinitesimal surface element (Fig. 2(b)) the magnetic flux density vector at a point is given by

$$
\bar{B}(\bar{r})=\frac{\mu_{o}}{4 \pi} \int_{A o} \frac{\bar{K}_{b}\left(\bar{r}_{o}\right) \times \hat{r}^{\prime}}{r^{\prime 2}} d A_{o}
$$

The vector $\bar{r}^{\prime}$ is the position vector of that point with respect to the location of the infinitesimal surface element. The unit vector along $\bar{r}^{\prime}$ is $\hat{r}^{\prime}$.

For a cylindrical magnet with axes system shown in Fig. 2(b)

$$
\bar{K}_{b}=M \hat{k} \times \hat{\varepsilon}_{\rho o}=M \hat{\varepsilon}_{\theta 0}
$$

Therefore,

$$
\bar{B}(\bar{r})=\frac{\mu_{o} M R}{4 \pi} \int_{z_{o}} \int_{0}^{2 \pi} \frac{\hat{\varepsilon}_{\theta o} \times \hat{r}^{\prime}}{r^{\prime 2}} d \theta_{o} d z_{o}
$$

From symmetry conditions it is evident that magnetic field perpendicular to a radial plane is zero. Moreover, the magnetic field distribution is identical for any radial plane. The coordinates $\theta_{0}$ and $z_{0}$ are measured with respect to this radial plane. The field at any point in this plane can be expressed as: -

$$
\bar{B}=\frac{\mu_{o} M R}{4 \pi} \int_{z_{o}} \int_{0}^{2 \pi} \frac{\left(-\sin \theta_{o} \hat{\varepsilon}_{r}+\cos \theta_{o} \hat{\varepsilon}_{\theta}\right) \times\left\{\left(r-R \cos \theta_{o}\right) \hat{\varepsilon}_{r}-R \sin \theta_{o} \hat{\varepsilon}_{\theta}+\left(z-z_{o}\right) \hat{k}\right\}}{\left\{\left(r-R \cos \theta_{o}\right)^{2}+\left(R \sin \theta_{o}\right)^{2}+\left(z-z_{o}\right)^{2}\right\}^{3 / 2}} d \theta_{o} d z_{o}
$$

From the above relation one obtains

$$
B_{r}=\frac{\mu_{o} M R}{4 \pi} \int_{z_{o}} \int_{0}^{2 \pi} \frac{\left(z-z_{o}\right) \cos \theta_{o}}{\left\{r^{2}+R^{2}+\left(z-z_{o}\right)^{2}+2 r R \cos \theta_{o}\right\}^{3 / 2}} d \theta_{o} d z_{o}
$$

It is difficult to evaluate the integral given by Eq. (5) analytically and a numerical integration scheme is adopted here for this purpose.

\subsubsection{Force on the magnet attached to the beam or structure}

The magnet attached to the beam is now replaced by a current distribution in the $\theta$ direction on a cylindrical surface (Fig. 3). The force on a line current placed in a magnet field is given by the following well-known relation:-

$$
\bar{F}=\int_{l} i d \bar{l} \times \bar{B}
$$

Here the current is denoted by $i$ and the symbol $d \bar{l}$ denotes an infinitesimal length vector of the line. Extending this formula for a surface current density $\bar{K}_{b}=\bar{M} \times \hat{n}$, the following relation is obtained

$$
\bar{F}=\int_{z} \int_{\theta}(\bar{M} \times \hat{n}) R d \theta d z \times \bar{B}=\int_{z} \int_{\theta}(-M \hat{k} \times \hat{n}) R d \theta d z \times \bar{B}=\iint_{z}\left(-M \hat{\varepsilon}_{\theta}\right) R d \theta d z \times\left(B_{r} \hat{\varepsilon}_{r}+B_{\theta} \hat{\varepsilon}_{\theta}\right)
$$

Here, both the permanent magnets are assumed to have the same axial magnetization $M$. The diameters $(2 R)$ of both the magnets are same and one is symmetrically placed on the top of the other maintaining a gap between them. From symmetry, for any infinitesimal area in the polar coordinate, the $\theta$-component of force is zero. The radial 


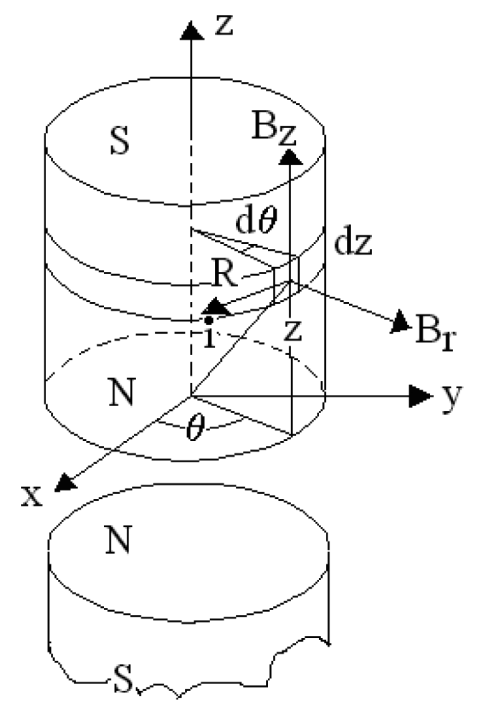

Fig. 3. Magnetic flux density due to one permanent magnet at the surface of the second one.

component of force on the magnet attached to the beam or structure becomes zero on integration in the $\theta$-direction. The $z$-component of the force can be expressed as shown below:-

$$
F_{z}=\int_{z} \int_{\theta} M B_{r} R d \theta d z=2 \pi M R \int_{z} B_{r} d z
$$

This integration is computed using the trapezoidal rule in one-dimension.

\subsection{Model of a piezoelectric stack}

Piezoelectric materials are used to convert electrical energy to mechanical energy and vice versa. The precise motion that results when an electric potential is applied to a piezoelectric material is of great practical interest in fine positioning (also called nano-positioning) applications. Piezoelectric materials are commercially available in different shapes and sizes. Stack is one of the most common shapes, which can undergo a free axial travel up to $300 \mu \mathrm{m}$ or even more in custom made cases. The symbol $d_{33}$ represents an important property of the piezoelectric material and in case of a stack, $n d_{33}$ represents static elongation of the stack per unit voltage, where the number of layers in the stack is $n$. The stack can be considered as a one-dimensional element and the stress-strain relation for the stack can be written as follows:-

$$
\sigma=E\left(\varepsilon-\frac{n d_{33}}{l_{s}} V\right)=E(\varepsilon-\alpha V)
$$

Where, the symbols $E$ and $V$ stand for elastic modulus of the material of the stack and the voltage applied across it.

$$
\alpha=\frac{n d_{33}}{l_{s}}
$$

The stress and strain in the stack are represented by symbols $\sigma$ and $\varepsilon$ respectively

The symbol $l_{s}$ stands for the length of the stack.

The piezoelectric stack is modelled as a bar element with uniformly distributed mass. The stress-strain relation represented by Eq. (9) can be tackled as a thermal strain problem [9], where the equivalent thermal coefficient of expansion is denoted by $\alpha$. The increase in temperature is equivalent to the applied voltage $V$. In the piezoelectric stack it is assumed that the elongation at any intermediate point can be obtained by linear interpolation of the elongations at the two ends. If the elongations of the two ends are denoted by the symbols $u_{1}$ and $u_{2}$, the equation of motion for an unloaded stack becomes [8] 


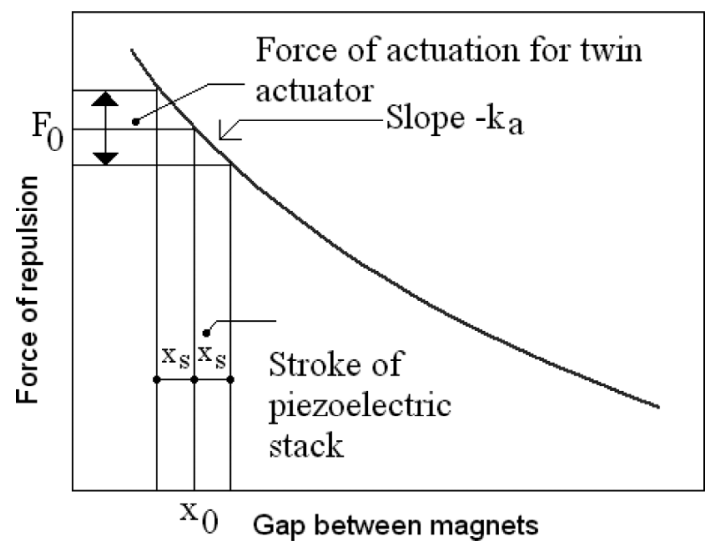

Fig. 4. An expected force-gap characteristics for two permanent magnets when the beam is kept fixed.

$$
\left[\begin{array}{cc}
\frac{m_{s}}{3} & 0 \\
0 & \frac{m_{s}}{3}
\end{array}\right]\left\{\begin{array}{l}
\ddot{u}_{1} \\
\ddot{u}_{2}
\end{array}\right\}+\left[\begin{array}{cc}
k_{s} & -k_{s} \\
-k_{s} & k_{s}
\end{array}\right]\left\{\begin{array}{l}
u_{1} \\
u_{2}
\end{array}\right\}=A E \alpha V\left\{\begin{array}{l}
1 \\
1
\end{array}\right\}
$$

If one end of the stack is kept fixed $\left(u_{2}=0\right)$ the above equation becomes

$$
\frac{m_{s}}{3} \ddot{u}_{1}+k_{s} u_{1}=A E \alpha V
$$

The total mass and the open loop stiffness of the stack are denoted by the symbols $m_{s}$ and $k_{s}$ respectively. The quantity $\frac{m_{s}}{3}$ appears due to the assumption of uniform mass distribution and that the displacement at any point on the stack can be obtained from a linear interpolation of those at the extreme ends. The cross-sectional area of the stack is represented by the symbol $A$.

$$
k_{s}=\frac{A E}{l_{s}}
$$

Using the relations

$$
\frac{m_{s}}{3} \ddot{u}_{1}+k_{s} u_{1}=k_{s} n d_{33} V
$$

The natural frequency of such a stack is

$$
\omega_{n}=\sqrt{\frac{3 k_{s}}{m_{s}}}
$$

At frequencies sufficiently lower than this natural frequency the dynamics of the stack can be neglected. If one denotes the elongation of the stack by the symbol $x_{s}$, then after neglecting the dynamic term in Eq. (12), he obtains

$$
x_{s}=u_{1}=\frac{A E \alpha V}{k_{s}}=n d_{33} V
$$

\subsection{The twin-actuator as a damper}

As shown in Fig. 1, actuation is performed using twin actuators. One actuator, with the piezoelectric stack and the permanent magnet mounted on it, acts from the bottom. The other actuator acts from the top. The beam has also got two permanent magnets attached to it, one at the bottom and the other at the top. The polarities of the magnets are so chosen that both the actuators repel the beam. When the gaps between the actuator magnet and the magnet attached to the beam are identical both at the top and the bottom, the net force on the beam is zero. Now, both the piezoelectric stacks are made to move up and down simultaneously. When the stack at the bottom moves upward it comes closer to the beam. But during its upward motion the stack at the top moves away from the beam. If the beam is kept fixed, the repulsive force in the upward direction from the bottom becomes higher than the repulsive force in the downward direction from the top. In this way a force starts acting on the beam. The nature of a typical force versus gap (between the actuator and the beam) characteristics for a single actuator is shown in Fig. 4. 


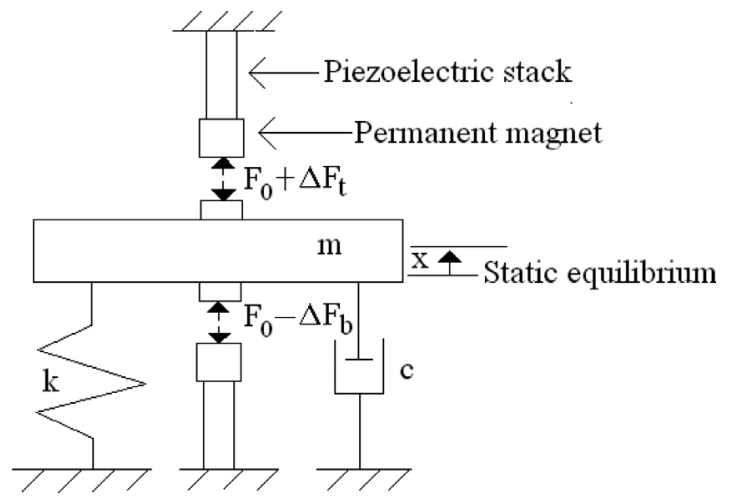

(a)

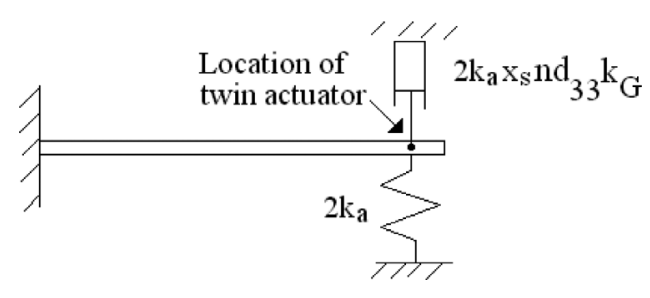

(b)

Fig. 5. (a). A single degree of freedom (SDOF) system with the proposed actuator; (b). Linear model of a beam with twin actuator.

\subsubsection{SDOF model}

For a single degree of freedom (SDOF) model of the beam or structure (Fig. 5(a)), the force of excitation is applied on the lumped mass $m$. If the lumped stiffness and damping values are represented by symbols $k$ and $c$. The external excitation on the beam is denoted by the symbol $f$. As the mass moves up by a displacement $x$, the repulsive force from the bottom actuator reduces by an amount $\Delta F_{b}$. The repulsive force from the top actuator increases by $\Delta F_{t}$. It is assumed that top and bottom actuator are perfectly identical and at the static equilibrium position they exert exactly equal and opposite force $F_{0}$ on the structure with equal gap of the beam from both the actuators.

$$
\begin{aligned}
& F_{t}=F_{0}+\Delta F_{t} \\
& F_{b}=F_{0}-\Delta F_{b}
\end{aligned}
$$

The equation of motion now becomes:-

$$
m \ddot{x}+c \dot{x}+k x=f+F_{b}-F_{t}=f-\left(\Delta F_{b}+\Delta F_{t}\right)
$$

One should note that when the beam moves up by a displacement $x$, the actuator can also be made to be move and the force $\left(\Delta F_{b}+\Delta F_{t}\right)$ depends on the net gaps between the actuators and the beam and not on the displacement $x$ of the beam alone.

When the mass $m_{M}$ of the magnet placed on the piezoelectric stack Eq. (14) is considered along with a reaction force acting on it, the equation of motion for the bottom actuator is as follows:-

$$
\left(m_{M}+\frac{m_{s}}{3}\right) \ddot{x}_{b s}+k_{s} x_{b s}=k_{s} n d_{33} V_{b}-\left(F_{0}-\Delta F_{b}\right)
$$

If the displacement is measured from the static equilibrium position of the actuator, the above equation becomes

$$
\left(m_{M}+\frac{m_{s}}{3}\right) \ddot{x}_{b s}+k_{s} x_{b s}=k_{s} n d_{33} V_{b}+\Delta F_{b}
$$

Similarly, the equation of motion for the top actuator

$$
\left(m_{M}+\frac{m_{s}}{3}\right) \ddot{x}_{t s}+k_{s} x_{t s}=k_{s} n d_{33} V_{t}-\Delta F_{t}
$$

The displacements $x_{b s}$ and $x_{t s}$ are in the direction of extension of the bottom and top actuators respectively (Fig. 1(b)).

Now, the idea is that, if one controls the voltages applied to the stacks and the consequential gaps between the the actuators and the beam in such a way that the net force on the beam $\left(\Delta F_{b}+\Delta F_{t}\right)$ becomes at least approximately proportional to the velocity of the beam at the location of the actuator, then the twin-actuator should work like a damper. In order to achieve this, the displacement of the stack is made proportional to the negative of the velocity of the beam at the location of the actuator. This is done in a feedback control loop (Fig. 1(a)). The displacement close to the location of the actuator is measured using a displacement sensor. This signal is differentiated and multiplied 
with a negative gain $-k_{G}$ in a controller. A power amplifier amplifies this signal and sends it to the piezoelectric stack at the bottom. The top stack receives a signal opposite in sign.

$$
V_{b}=-V_{t}=V=-k_{G} \dot{x}
$$

That a feedback in this form makes the actuator work like a damper becomes evident from the linearized and simplified equations discussed in the following sub-section 2.3.2.

Now, the force from an actuator is a non-linear function of net gap between that actuator and the beam. Equation (17) now becomes

$$
\begin{aligned}
& F_{b}=F_{b}\left(x_{0}-x_{b s}+x\right)=F_{b}\left(x_{0}\right)-\Delta F_{b} \\
& F_{t}=F_{t}\left(x_{0}-x_{t s}-x\right)=F_{t}\left(x_{0}\right)+\Delta F_{t}
\end{aligned}
$$

Since the actuators are assumed to be identical

$$
F_{b}\left(x_{0}\right)=F_{t}\left(x_{0}\right)=F_{0}
$$

The initial gap at the bottom and top is denoted by $x_{0}$ and the extensions of the bottom and top actuators are expressed using variables $x_{b s}$ and $x_{t s}$ respectively. The vibrating structure moves up by a displacement $x$ at the location of the actuators. The positive directions of these variables are shown in Fig. 1(b).

The non-linear functions presented above are obtained as tabular data from the magnetic analysis described in section 2.1. Since, both the bottom and top actuators are identical; the functional forms in Eq. (23) are also same.

Equations (18), (20), (21), (22) and (23) have to be simultaneously solved to ascertain the behaviour of the actuator as a damper.

\subsubsection{Linearization and simplification of the SDOF model}

The linearization starts from Eq. (23). The force-gap characteristics for both the actuators are assumed identical and known. The linearized force in the upward direction from the actuator at the bottom is given by,

$$
F_{b}=F_{b}\left(x_{0}-x_{b s}+x\right)=F_{0}-\Delta F_{b}=F_{0}+\left(\frac{\partial F_{b}}{\partial(g a p)}\right)_{x 0}\left(x-x_{b s}\right)=F_{0}-k_{a}\left(x-x_{b s}\right)
$$

Where, the symbol $F_{0}$ is defined in Eq. (23)

This linearization is valid when the magnitude of the displacement $\left(x-x_{b s}\right)$ is small compared to the initial gap $x_{0}$.

Where $k_{a}=-\left(\frac{\partial F_{b}}{\partial x}\right)_{x 0}$ is negative of the slope of the force-gap curve at the initial gap. As force of repulsion decreases with gap (Fig. 4), the quantity $k_{a}$ is positive.

Similarly, the force in the downward direction from the actuator at the top is

$$
F_{t}=F_{t}\left(x_{0}-x_{t s}-x\right)=F_{0}+\Delta F_{t}=F_{0}-\left(\frac{\partial F_{t}}{\partial(\text { gap })}\right)_{x 0}\left(x+x_{t s}\right)=F_{0}+k_{a}\left(x+x_{t s}\right)
$$

The magnitude of the displacement $\left(x+x_{t s}\right)$ is assumed to be small compared to the initial gap $x_{0}$. The linearized values of $\Delta F_{b}$ and $\Delta F_{t}$ are as follows:-

$$
\begin{aligned}
& \Delta F_{b}=k_{a}\left(x-x_{b s}\right) \\
& \Delta F_{t}=k_{a}\left(x+x_{t s}\right)
\end{aligned}
$$

It is already mentioned in section 2.2 that the dynamics of the piezoelectric stack can be neglected in the low frequency region, which is of interest in mechanical vibration. Moreover, since the stiffness of the stacks is very high, the forces $\Delta F_{b}$ and $\Delta F_{t}$ cannot have much effect on the displacements of the stack and can be dropped from Eqs (20) and (21). Under such simplifying assumptions, from Eqs (20), (21) and (22),

$$
\begin{aligned}
& x_{b s}=n d_{33} V_{b}=n d_{33} V=-n d_{33} k_{G} \dot{x}=x_{s} \\
& x_{t s}=-x_{b s}=n d_{33} k_{G} \dot{x}=-x_{s}
\end{aligned}
$$


Combining Eqs (19), (26) and (27), the final equation for the controlled structure becomes

$$
\begin{aligned}
& m \ddot{x}+c \dot{x}+k x=-2 k_{a} x-2 k_{a} n d_{33} k_{G} \dot{x}+f \\
& m \ddot{x}+\left(c+2 k_{a} n d_{33} k_{G}\right) \dot{x}+\left(k+2 k_{a}\right) x=f
\end{aligned}
$$

Therefore, for collocated sensor and twin-actuator, the twin-actuator adds a stiffness and a damper at the location of the actuator. For example, if one employs such a twin-actuator at the tip of a beam, it implies addition of a stiffness and damper there (Fig. 5(b)). For a given twin-actuator, the stiffness cannot be changed. However, the damping coefficient depends upon the control gain $k_{G}$.

\subsubsection{MDOF model}

In a multi-degree of freedom (MDOF) finite element model of the system let the $n \times 1$ vector $\{X\}$ contains the displacements of the beam or structure at its degrees of freedom. Let the actuator force is applied at the $j t h$ degree of freedom. If the mass, stiffness and damping matrices of sizes $n \times n$ are represented by the symbols $[M],[K]$ and $[D]$ respectively, the governing equation of motion for the system can be written as follows:-

$$
[M]\{\ddot{X}\}+[D]\{\dot{X}\}+[K]\{X\}=\left\{T_{e}\right\} f+\left\{T_{c}\right\}\left(-\Delta F_{b}-\Delta F_{t}\right)
$$

The right hand side of Eq. (30) is same as that of Eq. (18). The only difference now is that the beam has multiple degrees of freedom and the the twin actuator is associated with one of them.

The $n \times 1$ vector $\left\{T_{c}\right\}$ has all zero entries except for the $j t h$ row. This row has a unity entry. Similarly, $\left\{T_{e}\right\}$ has a unity entry only at that degree of freedom where the external excitation is applied.

If there are two identical exciters at $j t h$ and $k t h$ degrees of freedom, the column vector $\left\{T_{c}\right\}$ has to be replaced by a $n \times 2$ matrix $\left[T_{c}\right]$. The $j t h$ row of the first column and $k t h$ row of the second column contain unity entries. For the purpose of simplicity, in the following derivation, a single actuator has been considered.

In state space notations,

$$
\left\{\begin{array}{l}
\ddot{X} \\
\dot{X}
\end{array}\right\}=-\left[\begin{array}{l}
{[M]^{-1}[D][M]^{-1}[K]} \\
-[I]
\end{array} \begin{array}{l}
{[0]}
\end{array}\right\}\left\{\begin{array}{l}
\{\dot{X} \\
\{X\}
\end{array}\right\}+\left[\begin{array}{l}
{[M]^{-1}\left\{T_{e}\right\}[M]^{-1}\left\{T_{c}\right\}} \\
{[0]}
\end{array}\right]\left\{\begin{array}{l}
f \\
\left(-\Delta F_{b}-\Delta F_{t}\right)
\end{array}\right\}(31 \mathrm{a})
$$

Or,

$$
\{\dot{Z}\}=[A]\{Z\}+[B]\left\{\begin{array}{l}
f \\
\left(-\Delta F_{b}-\Delta F_{t}\right)
\end{array}\right\}
$$

The displacement of the beam at the location of the actuator can be obtained as

$$
x=[C]\{Z\}
$$

The equation for the bottom actuator

$$
\left\{\begin{array}{l}
\ddot{x}_{b s} \\
\dot{x}_{b s}
\end{array}\right\}=-\left[\begin{array}{cc}
2 \xi \bar{\omega}_{n} & \bar{\omega}_{n}^{2} \\
-1 & 0
\end{array}\right]\left\{\begin{array}{l}
\dot{x}_{b s} \\
x_{b s}
\end{array}\right\}+\left[\begin{array}{cc}
\bar{\omega}_{n}^{2} n d_{33} & 1 /\left(m_{s} / 3+m_{M}\right) \\
0 & 0
\end{array}\right]\left\{\begin{array}{l}
V_{b} \\
\Delta F_{b}
\end{array}\right\}
$$

Or,

$$
\left\{\dot{Z}_{b}\right\}=\left[A_{s}\right]\left\{Z_{b}\right\}+\left[B_{s}\right]\left\{\begin{array}{c}
V_{b} \\
\Delta F_{b}
\end{array}\right\}
$$

The equation for the top actuator

$$
\begin{aligned}
& \left\{\begin{array}{l}
\ddot{x}_{t s} \\
\dot{x}_{t s}
\end{array}\right\}=-\left[\begin{array}{cc}
2 \xi \bar{\omega}_{n} & \bar{\omega}_{n}^{2} \\
-1 & 0
\end{array}\right]\left\{\begin{array}{l}
\dot{x}_{t s} \\
x_{t s}
\end{array}\right\}+\left[\begin{array}{cc}
\bar{\omega}_{n}^{2} n d_{33} & -1 /\left(m_{s} / 3+m_{M}\right) \\
0 & 0
\end{array}\right]\left\{\begin{array}{c}
V_{t} \\
\Delta F_{t}
\end{array}\right\} \\
& \left\{\dot{Z}_{t}\right\}=\left[A_{s}\right]\left\{Z_{t}\right\}+\left[\bar{B}_{s}\right]\left\{\begin{array}{l}
V_{t} \\
\Delta F_{t}
\end{array}\right\}
\end{aligned}
$$




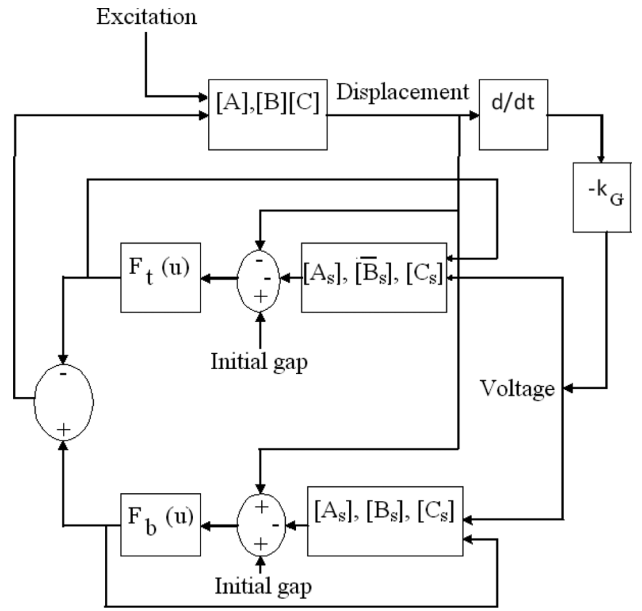

(a)

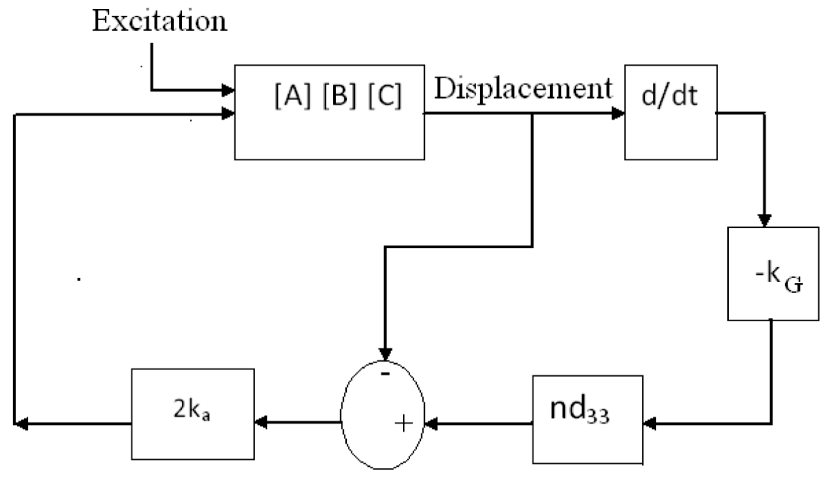

(b)

Fig. 6. (a). Block diagram showing the non-linear system; (b). Block diagram showing the linearized and simplified system.

Where, the symbol $\bar{\omega}_{n}$ indicates the natural frequency of a stack with a permanent magnet on it. The expression for $\bar{\omega}_{n}$ is

$$
\bar{\omega}_{n}=\sqrt{\frac{k_{s}}{m_{s} / 3+m_{M}}}
$$

In order to compute the response of the controlled system, Eqs (31), (33), (34), (22) and (23) have to be solved simultaneously. The block diagram for this model is shown in Fig. 6(a).

\subsubsection{Linearization and simplification of the MDOF model}

The force of actuation on the beam can be linearized as described in sub-section 2.3.2. The governing equation of the beam with the actuator Eq. (31a) is repeated here in a slightly different form.

$$
\left\{\begin{array}{l}
\ddot{X} \\
\dot{X}
\end{array}\right\}=-\left[\begin{array}{cc}
{[M]^{-1}[D]} & {[M]^{-1}[K]} \\
-[I] & {[0]}
\end{array}\right]\left\{\begin{array}{c}
\{\dot{X}\} \\
\{X\}
\end{array}\right\}+\left\{\begin{array}{c}
{[M]^{-1}\left\{T_{e}\right\}} \\
\{0\}
\end{array}\right\}\{f\}+\left\{\begin{array}{c}
{[M]^{-1}\left\{T_{c}\right\}} \\
\{0\}
\end{array}\right\}\left(-\Delta F_{b}-\Delta F_{t}\right)(36
$$

From Eqs (26) and (27),

$$
\left(-\Delta F_{b}-\Delta F_{t}\right)=-2 k_{a} x-2 k_{a} n d_{33} k_{G} \dot{x}
$$

The above linear Eqs (36) and (37) can be simultaneously solved as shown in the block diagram Fig. 6(b). However, a little manipulation shows that stiffness and a damping term are indeed added to the equation of motion.

$$
x=\left\{T_{c}\right\}^{T}\{X\}
$$

From Eqs (37) and (38)

$$
\left(-\Delta F_{b}-\Delta F_{t}\right)=-2 k_{a}\left\{T_{c}\right\}^{T}\{X\}-2 k_{a} n d_{33} k_{G}\left\{T_{c}\right\}^{T}\{\dot{X}\}
$$

Using Eq. (39) in Eq. (36)

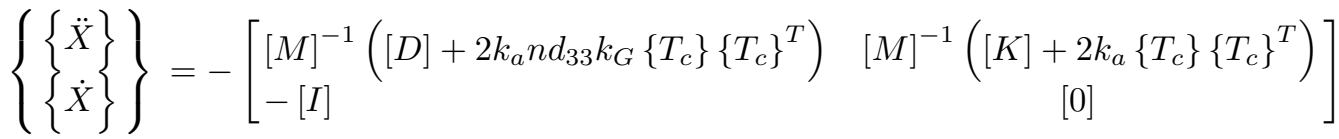

$$
\begin{aligned}
& \left\{\begin{array}{l}
\{\dot{X}\} \\
\{X\}
\end{array}\right\}+\left\{\begin{array}{l}
{[M]^{-1}\left\{T_{e}\right\}} \\
\{0\}
\end{array}\right\}\{f\}
\end{aligned}
$$




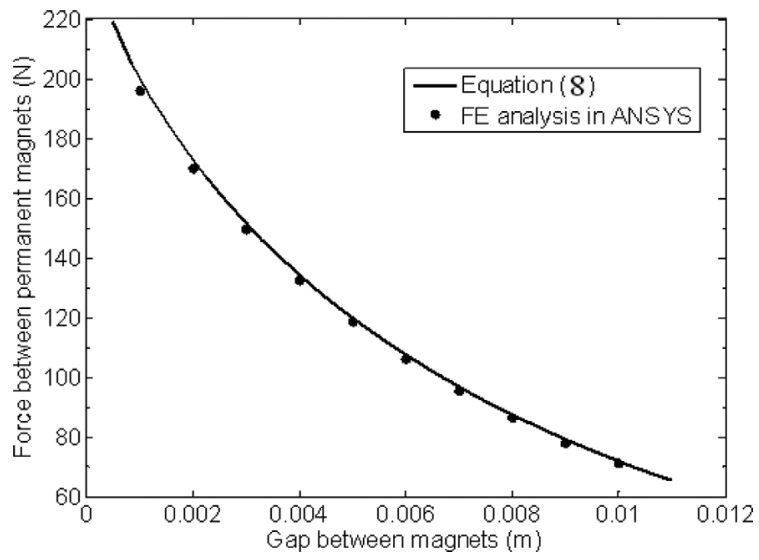

(a)

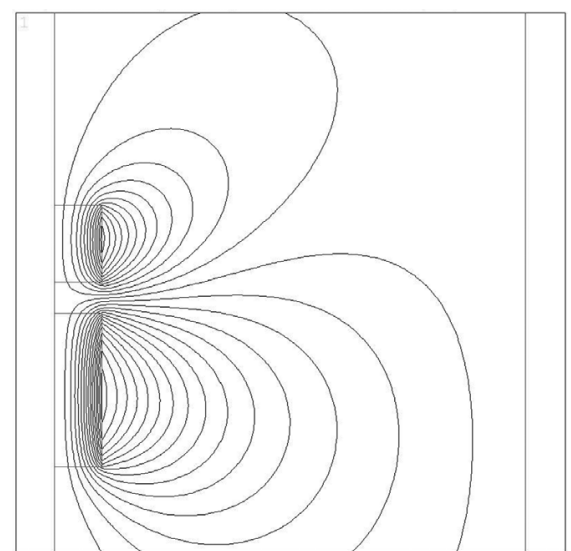

(b)

Fig. 7. (a). Force-gap plot for a single actuator; (b). Two-dimensional flux lines between two permanent magnets for a gap of $10 \mathrm{~mm}$ obtained from an axisymmetric finite element analysis in ANSYS.

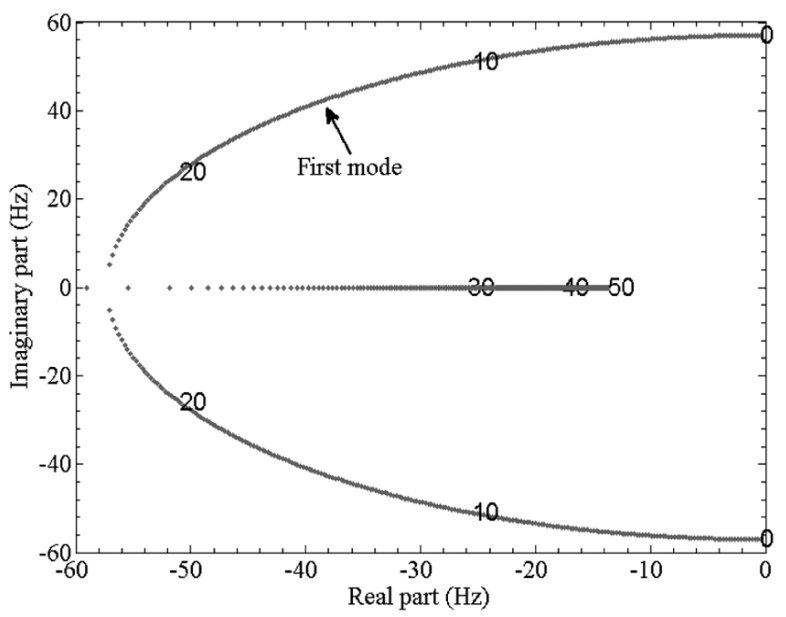

(a)

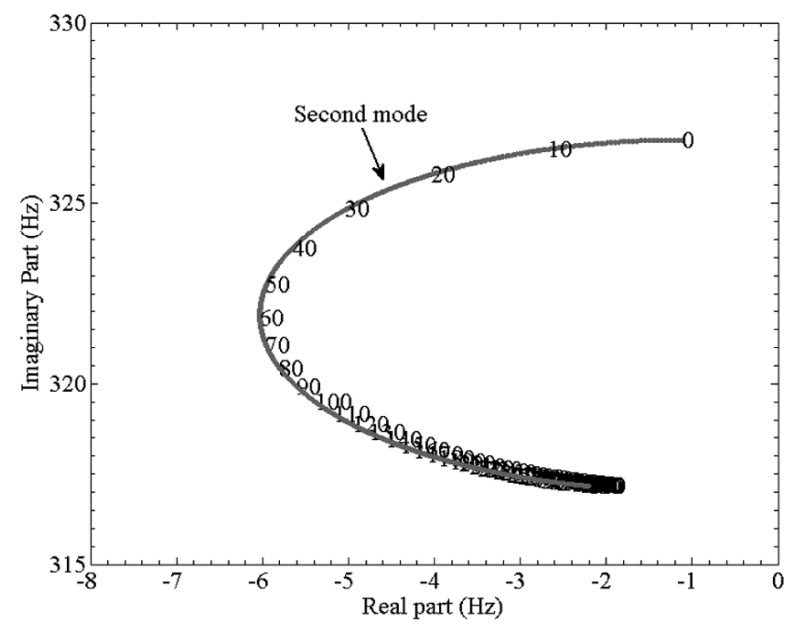

(b)

Fig. 8. Root locus diagram for the (a) first and (b) second modes obtained by varying the control gain.

The effective damping and stiffness matrices are denoted by symbols $[\bar{D}]$ and $[\bar{K}]$ respectively.

$$
\begin{aligned}
& {[\bar{D}]=[D]+2 k_{a} n d_{33} k_{G}\left\{T_{c}\right\}\left\{T_{c}\right\}^{T}} \\
& {[\bar{K}]=[K]+2 k_{a}\left\{T_{c}\right\}\left\{T_{c}\right\}^{T}}
\end{aligned}
$$

\section{Numerical study}

The force of a single actuator is computed using Eqs (5) and (8). The force is plotted in Fig. 7(a) as a function of gap between the beam and the actuator. The force is then verified with the result of an axisymmetric finite element analysis in commercial software ANSYS. The permanent magnet of the actuator and that attached to the beam are of lengths $50 \mathrm{~mm}$ and $25 \mathrm{~mm}$ respectively. The radii of both the magnets are $15 \mathrm{~mm}$. Neodymium (NdFeB) permanent magnets are nowadays the most widely used rare earth magnets. For such a magnet, a magnetization (magnetic 


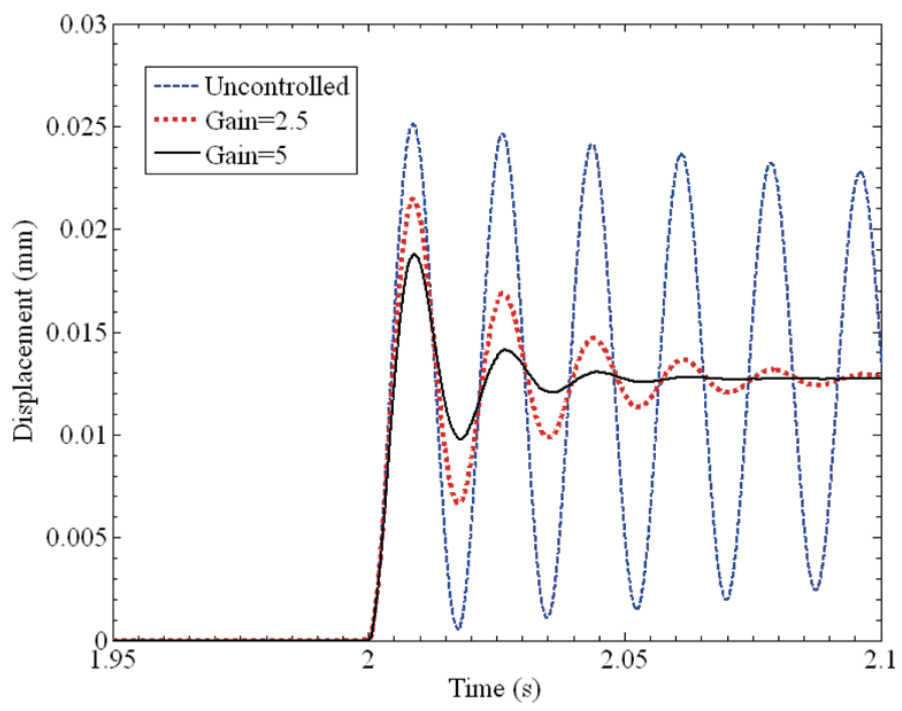

Fig. 9. Response of the uncontrolled and controlled system due to unit step excitation at $t=2 \mathrm{~s}$.

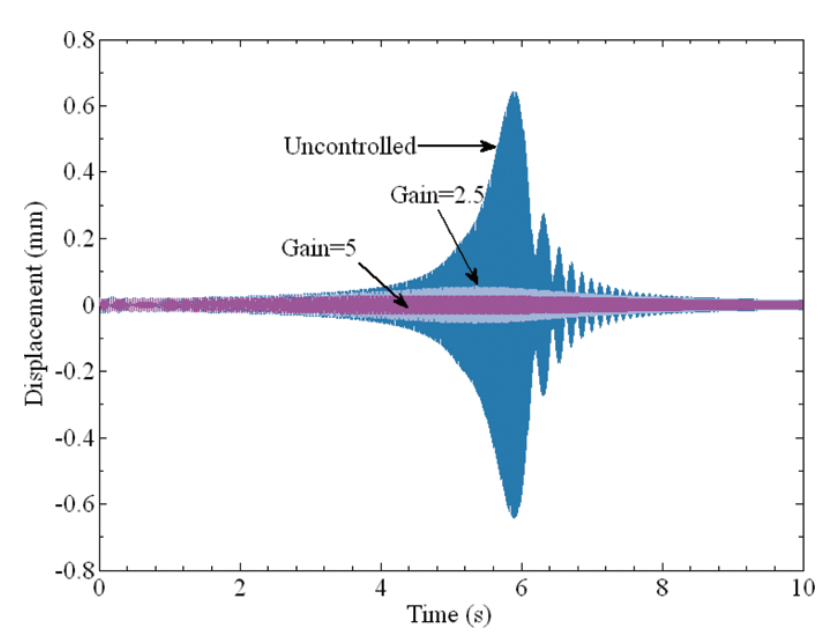

(a)

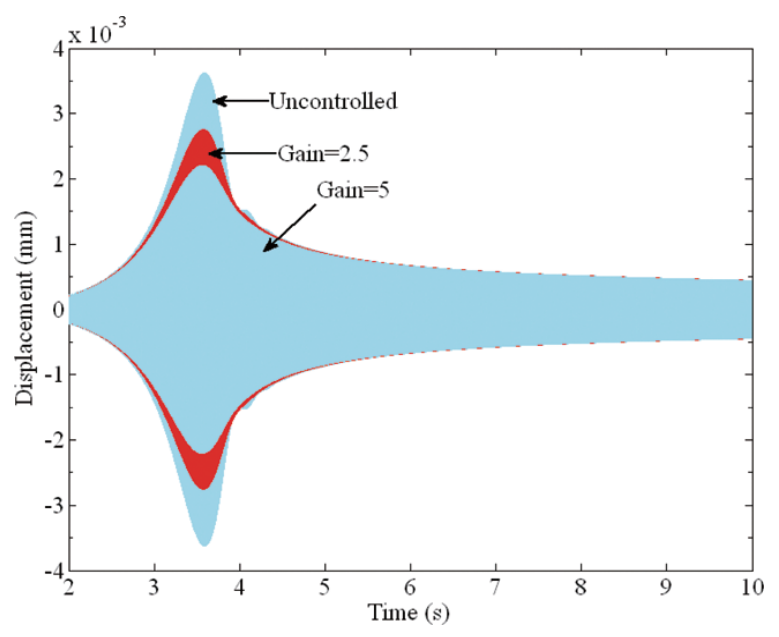

(b)

Fig. 10. (a). Response of the uncontrolled and controlled beam due to a swept sine signal of unit amplitude around its first resonance; (b). Response of the uncontrolled and controlled beam due to a swept sine signal of unit amplitude around its second resonance.

dipole moment per unit volume) of $\bar{M}=8 \times 10^{5} \hat{k} \mathrm{~A} / \mathrm{m}$ is considered. The relative permeability of Neodymium magnet is taken as $\mu_{r}=1$ in the finite element analysis. The magnetic flux lines obtained using ANSYS are shown in Fig. 7(b).

The piezoelectric stack selected for this purpose is close to one available in the catalogue of a well-known manufacturer in this field. The selected piezoelectric stack is $d_{s}=25 \mathrm{~mm}$ in diameter and $l_{s}=244 \mathrm{~mm}$ in length. It has a stiffness of $k_{s}=\frac{A E}{l_{s}}=54 \times 10^{3} \mathrm{~N} / \mathrm{mm}$ and can undergo a free static travel of $\delta_{s}=n d_{33} V=300 \mu \mathrm{m}$ when the voltage is increased from $0 \mathrm{~V}$ to $1000 \mathrm{~V}$. The equivalent coefficient of thermal expansion is $\alpha=1.2295 \times 10^{-6}$. The approximate mass of the stack is $m_{s}=164 \mathrm{gm}$ and the first natural frequency is at $f_{n s}=5 \mathrm{kHz}$. A permanent magnet of $m_{M}=270 \mathrm{gm}$ is placed on the piezoelectric stack. This reduces the natural frequency of the stack to $\bar{f}_{n}=2 \mathrm{kHz}$. According to the well-known thumb rule, up to one-tenth of its first natural frequency, i.e., $200 \mathrm{~Hz}$, the dynamics of the stack can be neglected. For higher frequencies the elongation-voltage characteristics can no longer be accurately determined from the relation $x_{s}=n d_{33} V$. Instead, the one-dimensional dynamic model of the 


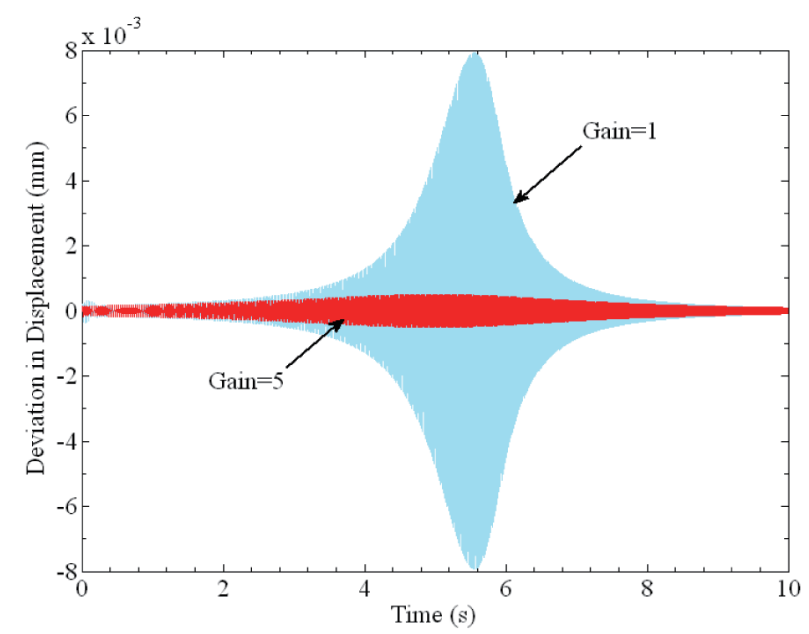

(a)

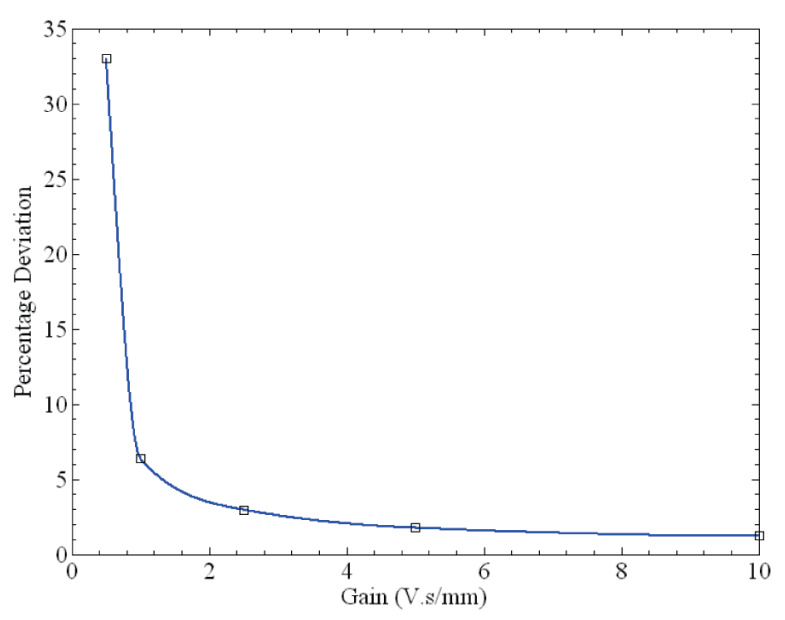

(b)

Fig. 11. (a) Deviation of results computed from non-linear and linear analyses and (b) maximum deviation as a percentage of maximum amplitude.

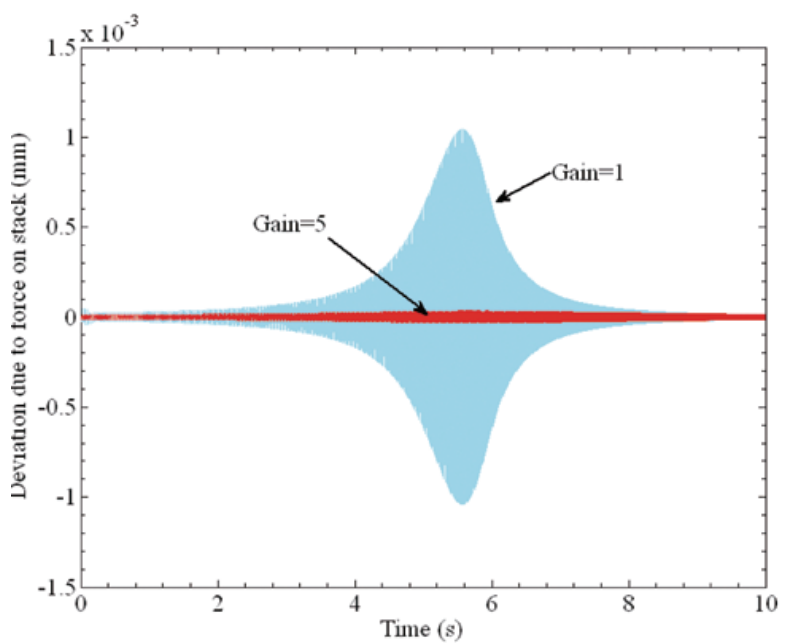

(a)

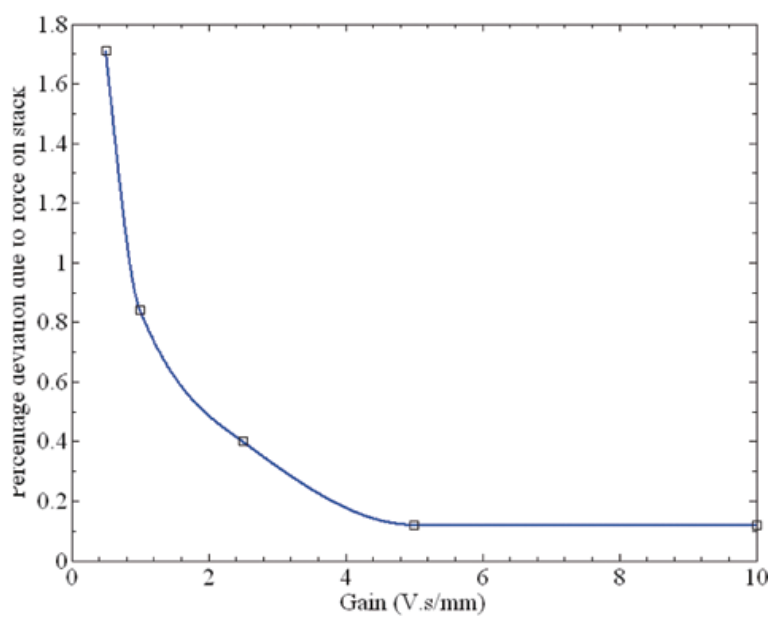

(b)

Fig. 12. (a) Deviation of results when forces on the stacks are neglected from the full non-linear analysis and (b) maximum deviation as a percentage of maximum amplitude.

actuator given by Eq. (12) now need be considered.

A ten-element finite element discretization of a steel cantilever beam is considered. The rotation degrees of freedom are removed using Guyan reduction technique. A small damping of the beam is considered in the form of Rayleigh damping. Both the non-linear system and the simplified model are integrated in time using SIMULINK. In SIMULINK, a user defined S-function is used to represent the beam model in state space form. The beam is $200 \mathrm{~mm}$ in length and $18 \mathrm{~mm} \times 5 \mathrm{~mm}$ in cross-section. The actuator is placed at the free end of the beam.

In the non-linear analysis (Fig. 6(a)) the force-displacement characteristics is modelled using tabular data. A small $0.5 \%$ viscous damping has been introduced in the stack model. The excitation is applied close to the actuator. The actuator is initially placed with a gap of $1 \mathrm{~mm}$ from the beam.

First, for the linear simplified representation (Fig. 6(b)) of the system with plant, actuator and controller, the eigenvalues are plotted as a function of the control gain $k_{G}$. The root locus plots for the first two modes are shown in Fig. 8. At a zero value of control gain, the first and second natural frequencies are approximately at $56 \mathrm{~Hz}$ and 


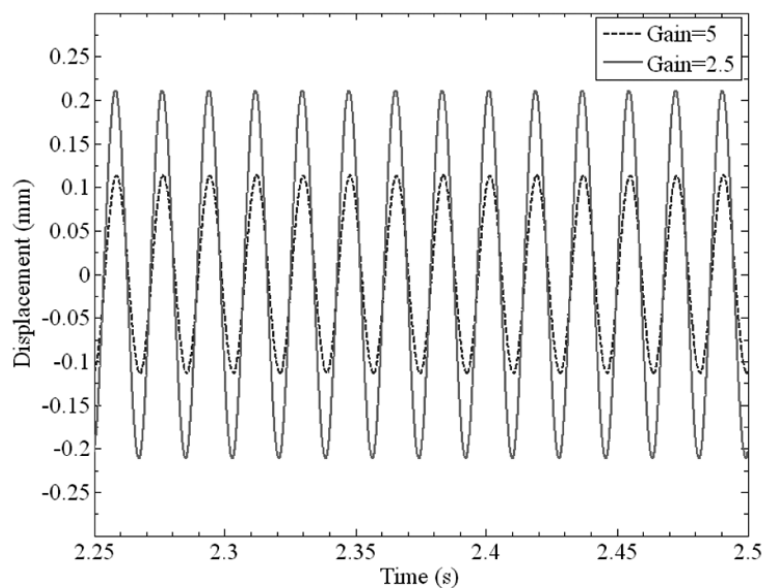

(a)

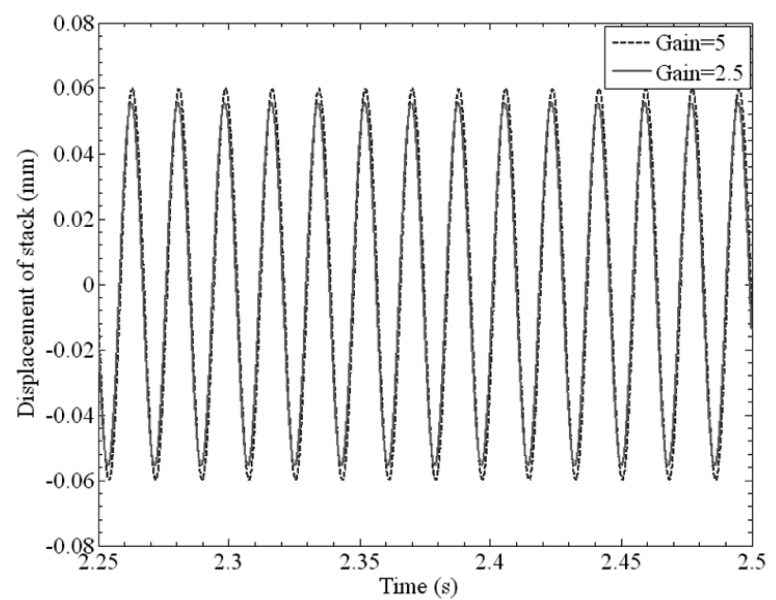

(b)

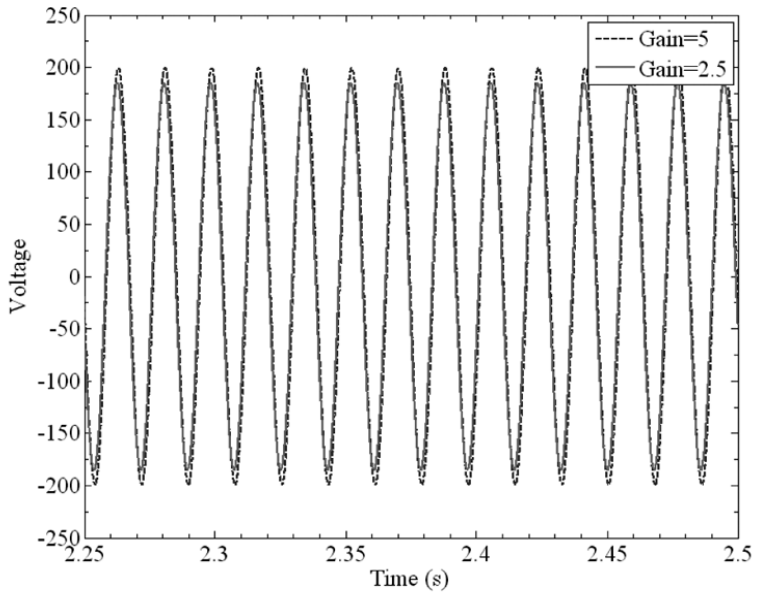

(b)

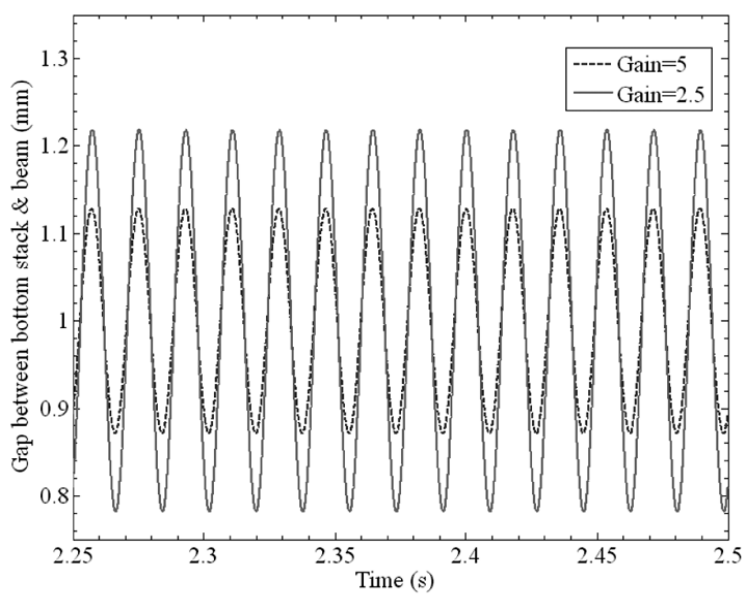

(d)

Fig. 13. (a) Displacement of the beam, (b) control voltage in the stack, (c) displacement of the bottom stack and (d) gap between the bottom actuator and the beam for a pure harmonic exciation of amplitude $4 N$ close to the first resonant frequency.

$327 \mathrm{~Hz}$. Both the root loci start from little left of the imaginary axis because of the damping of the material of the beam. This gap is very small for the first mode but can be observed on a closer examination. If the beam is approximated as a SDOF system having only the first mode, the real part of Fig. 8(a) equals the product of damping ratio $\xi$ and natural frequency $\Omega_{n}$. The imaginary part represents the damped natural frequency $\Omega_{d}=\Omega_{n} \sqrt{1-\xi^{2}}$. Initially as the gain increases from zero, the damping ratio increases. For a gain of a value 5, a SDOF approximation has a real part $\xi \omega_{n}=10$, which correspond to approximately $18 \%$ damping ratio.

The beam is first subjected to a unit step excitation applied at a time $t=2 \mathrm{~s}$. The response to the excitation is shown in Fig. 9 for the uncontrolled system and the controlled system with gains 2.5 and 5 . The vibration of the uncontrolled system reduces with time as there is a small damping present in the material of the beam. The controlled system quickly reaches its static equilibrium position without much of vibration.

The beam is then subjected to a swept sine signal. The amplitude of the signal is kept constant at a value of $1 \mathrm{~N}$. Two such swept sine signals are employed. For the first one the frequency centers around the first natural frequency of the beam and varies from $30 \mathrm{~Hz}$ to $80 \mathrm{~Hz}$ in a time span of $10 \mathrm{~s}$. During this excitation the beam passes through its first natural frequency. The response of the uncontrolled beam and the controlled one with three different gain values is presented in Fig. 10(a). The second swept sine signal varies from $310 \mathrm{~Hz}$ to $360 \mathrm{~Hz}$ in a time duration of $10 \mathrm{~s}$. This excitation signal excites the second resonance of the beam. The response is shown in Fig. 10(b). It is 
observed that the control gain is more effective in reducing the vibration at first resonance. This is already evident from the root locus diagram as for the first mode it goes much deeper in the left half of the complex plane.

All the above computations are performed using both the non-linear (Fig. 6(a)) and the linear models (Fig. 6(b)) of the system. The results from both the models almost exactly match and cannot be distinguished in a graphical representation. However, for the swept sine excitation, the displacement computed from the linear analysis is subtracted from that of the non-linear one and plotted in Fig. 11(a). The maximum deviation occurs at the maximum amplitude of the response. This is very well expected because as the displacement of the beam increases, the non-linearity becomes exceedingly pronounced. Therefore, a percentage deviation is computed as the ratio of the maximum deviation to the maximum amplitude of the non-linear simulation. This percentage deviation is plotted in Fig. 11(b). This figure clearly indicates that for a gain greater than 2 , the error due to linearization and simplification is less than a value of $4 \%$. This justifies the use of the linear simplified model in the present work.

Another interesting point is to study the effect of the force on the piezoelectric stack coming from the beam. In Eqs (20) and (21), the forces are designated by the symbols $\Delta F_{b}$ and $\Delta F_{t}$ for the bottom and top actuators respectively. Since the stiffness of the stacks is high it is expected that the deformation of the stacks due to such forces would be small. In order to have a quantitative assessment of this the non-linear simulation is done with and without force on the stack. This second option can easily be achieved in the SIMULINK model by making the force on the stack zero. The deviations are computed as is done in the previous case and plotted in Fig. 12(a). The maximum deviation is again at the location of maximum amplitude. The percentage deviation shown in Fig. 12(b) is indeed negligibly small.

Finally a pure harmonic excitation of amplitude $4 N$ and frequency close to the first natural frequency is applied to the beam. The responses for two different gain values are plotted in Fig. 13(a). The displacement amplitude for a gain of $5 \mathrm{Ns} / \mathrm{mm}$ is approximately half of that for a gain of $2.5 \mathrm{Ns} / \mathrm{mm}$. However, the voltage applied to the stack (Fig. 13(b)) approximately remains same for both the values of gain as it is the product of gain and velocity of vibration. The stack can accept a voltage within the range of 0-1000 V. In this work it is assumed that initially a static $500 \mathrm{~V}$ is applied to the stack. The control voltage shown in Fig. 13(c) is superposed on this static voltage. As the displacement of the stack, shown in Fig. 13(d), depends only on the voltage, they are also more or less same. Since the vibration is more for lower gain, the minimum gap between the actuator and the beam is less in this case.

\section{Conclusion}

The present work analyses the performance of a compact, non-contact type, active device for vibration damping. In the proposed device, control force on a beam is applied by imparting controlled motion to permanent magnets by using piezoelectric stacks. Since, the magnetic forces are essentially non-linear; a full non-linear simulation is performed. The proposed linear model has also been shown to work well except for extreme cases, where values of control gain are very small. The active damping device is tested for unit step excitation and swept sine excitation. Though the active damper works well for damping of second mode, it is particularly effective for control of vibration in first mode. The analysis in this work shows that the proposed actuator can be used with success for vibration control of beams and structures of small to medium sizes. A similar actuator with radially polarized permanent magnets may be explored for vibration damping of rotors.

\section{Acknowledgement}

This work started in Institute for Mechanics, University of Kassel during the first author's stay there as a research scientist (DAAD 425-FA).

\section{References}

[1] A. Chiba, T. Fukao, O. Ichikawa, M. Oshima, M. Takemoto and D. Dorell, Magnetic Bearings and Bearingless Drives, Newnes, Burlington, MA, 2005. 
[2] G. Genta, Dynamics of Rotating Systems, Springer, New York, 2004.

[3] D.J. Griffiths, 3rd Edition, Introduction to Electrodynamics, Prentice Hall of India, New Delhi, 2003.

[4] C. Kaletsch, R. Nordmann and B. Domes, Konstruktives und simulatives Ersetzen von passiven Quetschöldämpfer durch aktive Piezoaktoren für einen Flugtriebwerksniederdruckrotor, 8th Internantional Conference on Schwingungen in Rotierenden Maschinen, 23-25 Feb, Wien, Austria, 2009.

[5] T. Kreuzinger-Janik and H. Irretier, Experimental Modal Analysis - A Tool for Unbalance Identification of Rotating Machines, International Journal of Rotating Machinery 6(1) (2000), 11-18.

[6] T. Kreuzinger-Janik and H. Irretier, Unbalance Identification of Flexible Rotors based on Experimental Modal Analysis, 7th International Conference on Vibrations in Rotating Machinery, Nottingham, UK, 2000.

[7] C.W. Lee, Mechatronics in Rotating Machinery, IFToMM Proceedings of the 7th International Conference on Rotor Dynamics, 25th-28th Sep, Vienna, Austria, 2006.

[8] A. Nandi and S. Neogy, Performance Analysis of a Hybrid One-sided Magnetic Exciter mounted on a Piezoelectric Stack, Shock and Vibration 17 (2010), 205-215.

[9] A. Preumont, Mechatronics - Dynamics of Electromechanical and Piezoelectric Systems, Springer, Netherlands, 2005.

[10] P.M. Przybylowicz, Active stabilisation of a rigid rotor by a piezoelectrically controlled mobile journal bearing system, IFToMM Proceedings of the 6th International Conference on Rotor Dynamics, 30th Sep 30 to 4th Oct, 2002, Sydney, Australia.

[11] G. Schweitzer, H. Bleuler and A. Traxler, Active Magnetic Bearings: Basics, Properties and Applications of Active Magnetic Bearings, Authors' Reprint, Zurich, 2003.

[12] R.C. Simoes, J. Der Hagopian, J. Mahfoud and V. Steffen, Jr., About the Implementation of an Optimal Modal Controller in Flexible Rotor by Using Piezoelectric Actuators, IFToMM Proceedings of the 7th International Conference on Rotor Dynamics, 25th-28th Sep, Vienna, Austria, 2006.

[13] D. Strohschein and H. Irretier, Modellbildung eines biegeelastischen Rotors mit aktiver Dämpfung, 8th International Conference on Schwingungen in Rotierenden Maschinen, 23-25 Feb, Wien, Austria, 2009. 

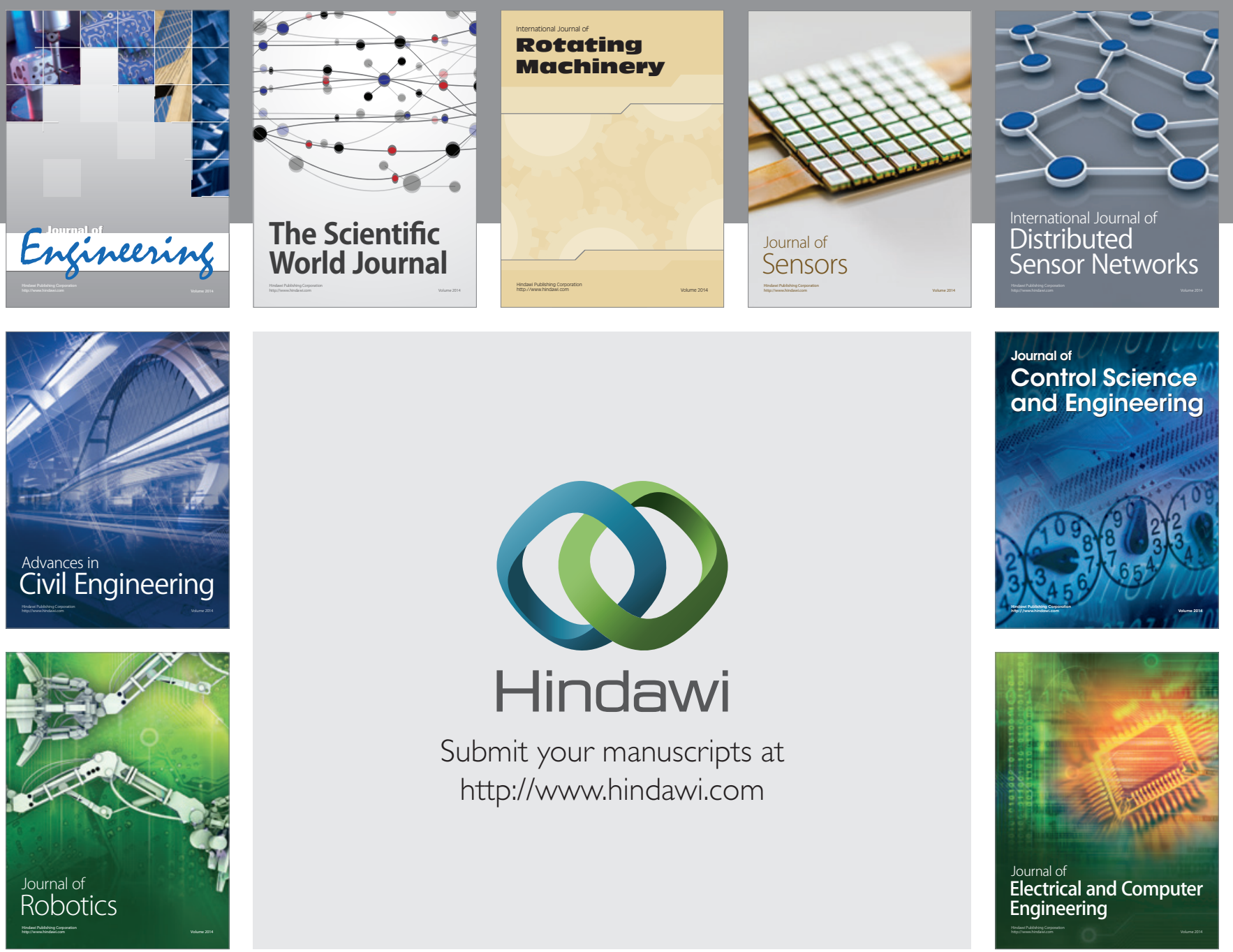

Submit your manuscripts at

http://www.hindawi.com
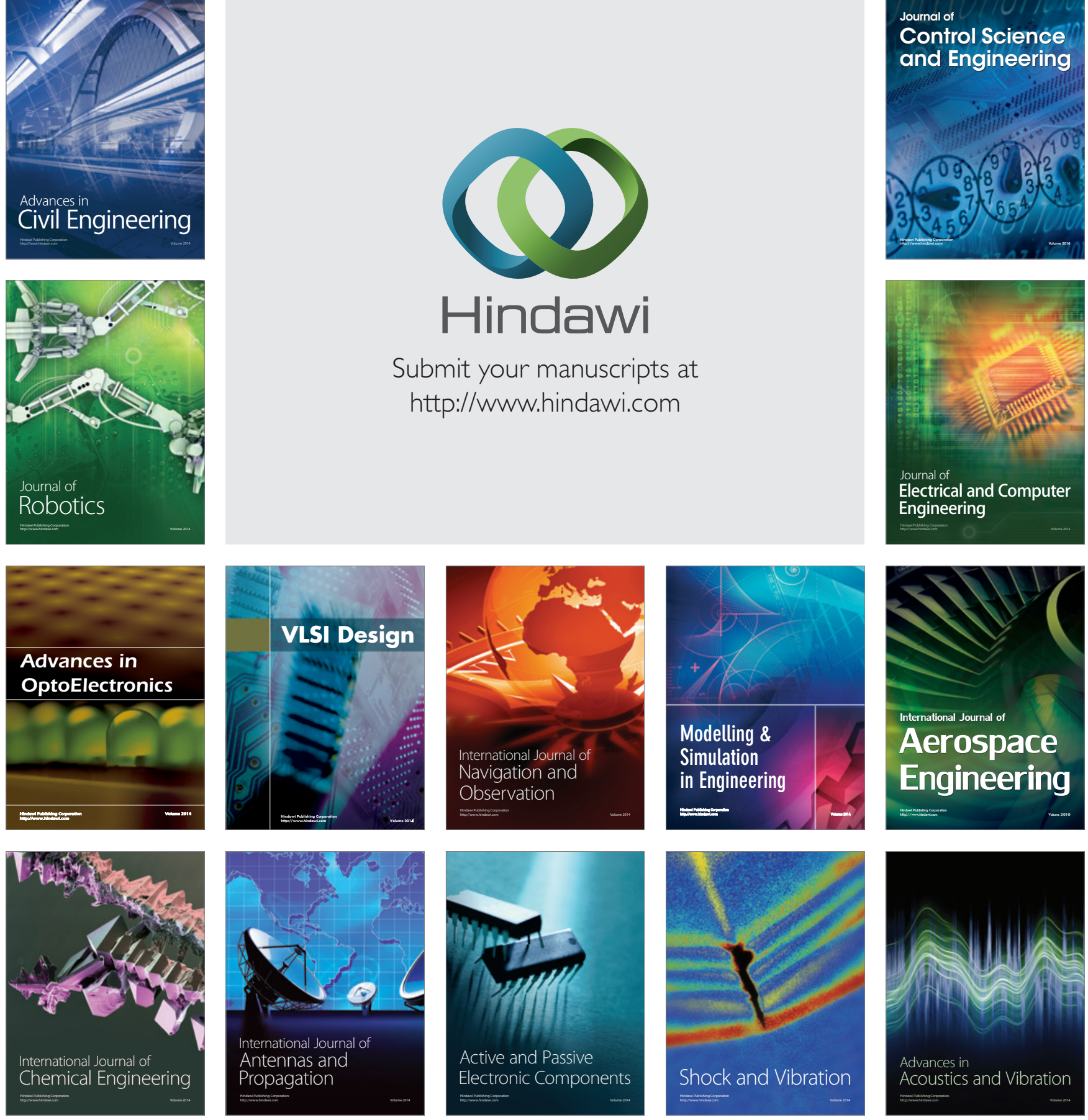